\title{
Effect of Monopole Field on the Non-Spherical Gravitational Collapse of Radiating Dyon Solution.
}

\author{
${ }^{1}$ S.S.Zade, ${ }^{2 *}$ C.S.Khodre, ${ }^{3}$ K.D.Patil \\ ${ }^{l}$ Department of Mathematics, J.B. College of Science, Wardha (M.S.), India \\ ${ }^{2}$ Department of Mathematics, S.D.College of Engineering, Selukate, Wardha (M.S.), India \\ ${ }^{3}$ Department of Mathematics, B.D.College of Engineering, Sevagram, Wardha (M.S.), India
}

\begin{abstract}
We study the effect of monopole field on gravitational collapse of radiating Dyon solution in nonspherical topology. Our main aim is to investigate the possibility of cosmic censorship violation in nonspherical gravitational collapse of radiating dyon solution in the presence of monopole field. It is shown that the final outcome of the collapse depends sensitively on the electric and magnetic charge parameters. The graphs with respect to the charge and monopole field show that the singularity is to be naked. It is an interesting feature emerges that the strength of naked singularity is weak curvature singularity. Though the naked singularity is weak one may conclude that the non-spherical gravitational collapse of monopole radiating Dyon solution violets the $\mathrm{CCH}$.
\end{abstract}

Keywords: Cosmic censorship, gravitational collapse, naked singularity, radiating Dyon solution.

\section{Introduction}

In the past forty years, there have been extensive studies on gravitational collapse investigating the nature of singularities. The study of gravitational collapse of spherically symmetric spacetimes has led to many examples of naked singularities.[1- 10] Most of the studies concentrated on the aim to find light rays emanating from the central singularity and escaping the Schwarzschild an trapped surface at least locally. In order to study the nature of the singularity, global behaviour of radial null geodesics must be studied in full generality.

The cosmic censorship hypothesis $(\mathrm{CCH})$, proposed by Penrose [11] states that the singularities formed in gravitational collapse of physically reasonable matter cannot be observed. Many researchers have attempted to give precise reformulation to this hypothesis, but neither proof nor mathematical formulation for this hypothesis is available so far. Almost all research papers published on $\mathrm{CCH}$ till date are limited to the collapse in spherically symmetric spacetimes. Of course, spherical symmetry is a characteristic feature of many solutions of Einstein's field equations of general relativity, but to prove (or disprove) the $\mathrm{CCH}$, it is essential to study the gravitational collapse in non-spherical case as well.

The singularity theorems (Homogeneous dust ball [12]) state that there exist space-time singularities in generic gravitational collapse. At singularities the smoothness of the spacetime metric is lost. More precisely, a singularity is not regarded as a point in the space-time manifold but one in the boundary of spacetime (Inhomogeneous dust ball [13]). Classical physics cannot be applied to space-time singularities because classical physics implicitly assumes the smoothness of space-time, where and hereafter refer to all physics which do not contain quantum gravity as classical physics. This implies the limitation of classical physics [14].

In spite of this limitation, it is possible to have some kind of future predictability using only classical physics if all singularities are hidden from physically relevant regions. Penrose [11] conjectured that a system which evolves, according to classical general relativity with reasonable equations of state, from generic non-singular initial data on a suitable Cauchy hypersurface, does not develop any space-time singularity which is visible from infinity. This conjecture is actually the weak version of censorship. If this conjecture is true, we can predict the whole evolution using classical physics for the space-time region outside black holes. The strong version [15] states that a physically reasonable classical space-time is globally hyperbolic. This conjecture claims that classical physics can predict the evolution of the whole space-time. A singularity which is censored by the strong version of censorship is called a naked singularity. A naked singularity which is censored by the weak version is called a globally naked singularity. A naked singularity which is not a globally naked singularity is called a locally naked singularity.

It is clear though, that the non-spherical collapse scenario is rather complex to understand, and recourse to the numerical simulations of evolving collapse models may greatly enhance our understanding on possible collapse final states in this case. In such a context, the numerical calculations of Shapiro and Teukolsky (1991) indicated conformity with the hoop conjecture. They evolved collissionless gas spheroids in full general relativity which collapse in all cases to singularities. When the spheroid is sufficiently compact a black hole may form, but when the semimajor axis of the spheroid is sufficiently large, a spindle singularity forms without an apparent horizon forming. This gives rise to the possibility of occurrence of naked singularities in collapse of 
finite systems in asymptotically flat spacetimes which violate weak cosmic censorship but are in accordance with the hoop conjecture [16].

The issue of the stability of naked singularity has recently attracted some attention in the literature [17]. The example leading to unstable naked singularities cannot be considered as physically realistic counter example to $\mathrm{CCH}$. Keeping this view in mind, we would like to investigate whether the naked singularities formed in the non-spherical gravitational collapse of monopole radiating Dyon solution are stable when one makes from spherical to non-spherical spacetimes. Also we would like to see whether the non-spherical gravitational collapse of monopole radiating Dyon solution contradicts the $\mathrm{CCH}$.

\section{Non-Spherically Symmetric Radiating Dyon Spacetime}

To study the non-spherical collapse of radiating dyon solution, firstly we shall give a brief discussion on general non-spherically symmetric spacetime.

The line element for toroidal (cylindrical or planar) spacetime is given by $[18,19]$

$$
d s^{2}=-\left[\alpha^{2} r^{2}-\frac{q m(v, r)}{r}\right] d v^{2}+2 d v d r+r^{2}\left(d \theta^{2}+d \emptyset^{2}\right)
$$

Where $v$ is an advanced Eddington time coordinate and $\alpha$ is given by the relation $3 \alpha^{2}=-\Lambda . m(v, r)$ is the gravitational mass of star whose radius is $r$ unit. Coordinates $\theta, \emptyset$ describe the two dimensional zero-curvature space generated by the two dimensional commutative Lie group $\mathrm{G}_{2}$ of isometrics [19], we write the topology of two dimensional spaces for metric (1) can be written as:

Topology of Toroidal model is $S \times S$, Cylindrical model has topology $R \times S$ and for planer Symmetrical model has $R \times R$. Ranges for $\theta$ and $\emptyset$ in Toroidal, Cylindrical and Planar models are given by
(i) Toroidal:
$0 \leq \theta<2 \pi$
$0 \leq \emptyset<2 \pi$
(ii) Cylindrical:
$-\infty<\theta<\infty$
$0 \leq \emptyset<2 \pi$
(iii) Planar:
$-\infty<\theta<\infty$
$-\infty<\emptyset<\infty$

Depending upon the topology of the two-dimensional space, parameter $q$ has different values. In the case of toroidal model, $m(v, r)$ is mass and parameter $q=\frac{2}{\pi}$, for the Cylindrical case $m(v, r)$ is mass per unit length and $q=\frac{4}{\alpha}$ and for Planar symmetrical model $m(v, r)$ is mass per unit area and $=\frac{2}{\alpha^{2}}$. The values of parameter $q$ are taken from Arnowitt-Deser-Misner (ADM) masses of the corresponding static black hole [19].

The mass function of radiating Dyon solution as

$$
q m(v, r)=q M(v)+a r-\left(\frac{q^{2} H_{e}^{2}(v)+q^{2} H_{m}^{2}(v)}{r}\right)
$$

where $M(v)$ is the usual Vaidya mass and $H_{e}^{2}(v)$ and $H_{m}^{2}(v)$ are electric charge and magnetic charge respectively.

In general equation (2) does not yield analytic solution. If however consider $H_{e}^{2}(v) \propto v^{2}$ and $H_{m}^{2}(v) \propto v^{2}$, then equation (2) becomes homogeneous and can be solved in terms of elementary functions. To get the analytic solution of equation (2) we take

$$
\begin{aligned}
q^{2} H_{e}^{2}(v) & =\delta^{2} v^{2} \\
q^{2} H_{m}^{2}(v) & =\mu^{2} v^{2}
\end{aligned}
$$

and

$$
q M(v)=\lambda v
$$

Then the mass function (2) becomes

$$
q m(v, r)=\lambda v+a r-\left(\frac{\delta^{2} v^{2}+\mu^{2} v^{2}}{r}\right)
$$

substituting this mass function in equation (1), we get

$$
d s^{2}=-\left[\alpha^{2} r^{2}-\frac{\lambda v}{r}-a+\frac{\delta^{2} v^{2}}{r^{2}}+\frac{\mu^{2} v^{2}}{r^{2}}\right] d v^{2}+2 d v d r+r^{2}\left(d \theta^{2}+d \emptyset^{2}\right)
$$

From the metric (1) and using mass function equation (6) we can write the non-zero metric components as given below

$$
\begin{array}{lll}
g_{00}=-\left(\alpha^{2} r^{2}-\frac{\lambda v}{r}-a+\frac{\delta^{2} v^{2}}{r^{2}}+\frac{\mu^{2} v^{2}}{r^{2}}\right), & & g_{01}=g_{10}=1 \\
g_{22}=g_{33}=r^{2}, & & g_{0}^{0}=g_{1}^{1}=g_{2}^{2}=1 \\
g^{11}=\alpha^{2} r^{2}-\frac{\lambda v}{r}-a+\frac{\delta^{2} v^{2}}{r^{2}}+\frac{\mu^{2} v^{2}}{r^{2}}, & & g^{22}=g^{33}=\frac{1}{r^{2}}
\end{array}
$$

For the metric (1) the non-vanishing christoffel symbols of second kind can be calculated using mass function equation (6) are

$$
\begin{aligned}
& \Gamma_{00}^{0}=\alpha^{2} r+\frac{\lambda v}{2 r^{2}}, \quad \Gamma_{22}^{0}=\Gamma_{33}^{0}=-r \\
& \Gamma_{00}^{1}=\lambda-\alpha^{2} a r-\alpha^{4} r^{3}+\frac{a^{2}}{r}-\frac{\delta^{2} v}{r^{2}}-\frac{\mu^{2} v}{r^{2}}+\frac{3 a \lambda v}{2 r^{2}}+\frac{\lambda^{2} v^{2}}{2 r^{3}}-\frac{a \delta^{2} v^{2}}{r^{3}}-\frac{a \mu^{2} v^{2}}{r^{3}}-\frac{\lambda \delta^{2} v^{3}}{2 r^{4}}-\frac{\lambda \mu^{2} v^{3}}{2 r^{4}} \\
& \Gamma_{01}^{1}=-\alpha^{2} r-\frac{\lambda v}{2 r^{2}}+\frac{\delta^{2} v^{2}}{r^{3}}+\frac{\mu^{2} v^{2}}{r^{3}}
\end{aligned}
$$




$$
\begin{aligned}
& \Gamma_{22}^{1}=\Gamma_{33}^{1}=a r+\lambda v-\alpha^{2} r^{3}-\frac{\delta^{2} v^{2}}{r}-\frac{\mu^{2} v^{2}}{r} \\
& \Gamma_{12}^{2}=\Gamma_{13}^{3}=\frac{1}{r}
\end{aligned}
$$

Now using the general formula, we find the components of Ricci tensor as given below

$$
\begin{aligned}
& R_{00}= 3 \alpha^{2}\left(r^{2}-1\right)+\frac{\lambda}{r^{2}}-\frac{3 \alpha^{2} \lambda v}{r}+4 \alpha^{2}\left(\frac{\delta^{2} v^{2}+\mu^{2} v^{2}}{r^{2}}\right)+\left(\frac{\delta^{2} v^{2}+\mu^{2} v^{2}}{r^{3}}\right)^{2}-2\left(\frac{\delta^{2} v+\mu^{2} v}{r^{3}}\right)-a\left(\frac{\delta^{2} v^{2}+\mu^{2} v^{2}}{r^{4}}\right)- \\
& \lambda\left(\frac{\delta^{2} v^{3}+\mu^{2} v^{3}}{r^{5}}\right) \\
& R_{01}=-3 \alpha^{2}-\left(\frac{\delta^{2} v^{2}+\mu^{2} v^{2}}{r^{4}}\right) \\
& R_{22}= R_{33}=a-3 \alpha^{2} r^{2}+\left(\frac{\delta^{2} v^{2}+\mu^{2} v^{2}}{r^{2}}\right) \\
& \text { and } R_{\mu v}=0 \text { otherwise }
\end{aligned}
$$

The Ricci Scalar is given by

$$
R=R_{i j} g^{i j}
$$

i.e. $\quad R=-12 \alpha^{2}+\frac{2 a}{r^{2}}$

Non-vanishing components of the Einstein's tensor are

We find that

$$
G_{j}^{i}=R_{j}^{i}-\frac{1}{2} R g_{j}^{i}
$$

$$
\begin{aligned}
& G_{0}^{0}=G_{1}^{1}=3 \alpha^{2}-\frac{a}{r^{2}}-\frac{\delta^{2} v^{2}}{r^{4}}-\frac{\mu^{2} v^{2}}{r^{4}} \\
& G_{2}^{2}=G_{3}^{3}=3 \alpha^{2}+\frac{\delta^{2} v^{2}}{r^{4}}+\frac{\mu^{2} v^{2}}{r^{4}} \\
& G_{0}^{1}=\frac{\lambda}{r^{2}}-\frac{2 \delta^{2} v}{r^{3}}-\frac{2 \mu^{2} v}{r^{3}}
\end{aligned}
$$

Therefore the Einstein field equations are

$$
G_{\mu v}=k T_{\mu v}
$$

where $G_{\mu \nu}$ is Einstein tensor, $k$ is a gravitational constant and $T_{\mu \nu}$ is energy momentum tensor of radiation and null dust fluid in Vaidya solution is of the form [20].

$$
T_{\mu v}=T_{\mu v}^{(n)}+T_{\mu v}^{(m)}
$$

where $\quad T_{\mu v}^{(n)}=\sigma l_{\mu} l_{v}, \quad T_{\mu v}^{(m)}=(\rho+p)\left(l_{\mu} \eta_{v}+l_{v} \eta_{\mu}\right)+p g_{\mu v}$

We have consider null vectors $l_{\mu}, \eta_{\mu}$ such that

$$
\begin{aligned}
& l_{\mu}=\delta_{\mu}^{0}, \quad \eta_{\mu}=\frac{1}{2}\left[\alpha^{2}-\frac{q \dot{m}}{r}\right] \delta_{\mu}^{0}-\delta_{\mu}^{1} \\
& l_{\lambda} l^{\lambda}=\eta_{\lambda} \eta^{\lambda}=0, \quad l_{\lambda} \eta^{\lambda}=-1
\end{aligned}
$$

Using the equations (13)-(19) we can write the expressions for $\sigma, \rho$ and $p$ as

$$
\begin{aligned}
& \sigma=\frac{\lambda}{k r^{2}}-\frac{2 \delta^{2} v}{k r^{3}}-\frac{2 \mu^{2} v}{k r^{3}} \\
& \rho=\frac{a}{k r^{2}}+\frac{\delta^{2} v^{2}}{k r^{4}}+\frac{\mu^{2} v^{2}}{k r^{4}}-\frac{3 \alpha^{2}}{k} \\
& p=\frac{3 \alpha^{2}}{k}+\frac{\delta^{2} v^{2}}{k r^{4}}+\frac{\mu^{2} v^{2}}{k r^{4}}
\end{aligned}
$$

Here $\rho, p$ are energy density and pressure, while $\sigma$ is the density of the Vaidya null radiation.

Energy conditions for such type of fluids are given by $[18,21]$

(I) The weak and strong energy conditions

$$
\sigma>0, \quad \rho \geq 0, \quad p \geq 0
$$

(II) The dominant energy condition

$$
\sigma>0, \quad \rho \geq p \geq 0
$$

with the proper choice of mass function $m(v, r)$, above condition can be satisfied.

Substituting the mass function equation (6) into equation (1), we get

$$
d s^{2}=-\left[\alpha^{2} r^{2}-\frac{\lambda v}{r}-a+\frac{\delta^{2} v^{2}}{r^{2}}+\frac{\mu^{2} v^{2}}{r^{2}}\right] d v^{2}+2 d v d r+r^{2}\left(d \theta^{2}+d \emptyset^{2}\right)
$$

\section{Nature Of The Singularity}

To study the nature of singularities may develop in gravitational collapse, either spherical or otherwise. However, if they are not either generic or stable in some suitable sence, then they may not be necessarily physical relevant [16] .Hence it would be interesting to investigate whether the gravitational collapse of nonspherical radiating dyon solution with monopole field could yield naked singularity under the influence of the composite field produced by electric and magnetic charges. Thus to investigate the structure of the collapse, we need to consider the radial null geodesics defined by $d s^{2}=0,\left(k^{\theta}=k^{\varnothing}=0\right)$ [10].

The equation for outgoing radial null geodesics for metric (25) is given by 


$$
\frac{d r}{d v}=\frac{1}{2}\left(\alpha^{2} r^{2}-\frac{\lambda v}{r}-a+\frac{\delta^{2} v^{2}}{r^{2}}+\frac{\mu^{2} v^{2}}{r^{2}}\right)
$$

It can be observed from equation (26) that this equation has a singularity at $r \rightarrow 0, v \rightarrow 0$. In order to classify the radial and non-radial outgoing non-space like geodesics terminating at this singularity in the past, consider $X$ is a self-similar variable defined by $X=\frac{v}{r}$ along a singular geodesic at the singularity is approached $[22,23,24]$. Let,

$$
\begin{aligned}
& X_{0}=\lim _{\substack{v \rightarrow 0 \\
r \rightarrow 0}} X=\lim _{\substack{v \rightarrow 0 \\
r \rightarrow 0}} \frac{v}{r} \\
& X_{0}=\lim _{\substack{v \rightarrow 0 \\
r \rightarrow 0}} \frac{d v}{d r}
\end{aligned}
$$

then equation (26) can be written as

$$
X_{0}=\frac{2}{-a-\lambda X_{0}+\delta^{2} X_{0}^{2}+\mu^{2} X_{0}^{2}}
$$

i.e. $\left(\mu^{2}+\delta^{2}\right) X_{0}^{3}-\lambda X_{0}^{2}-a X_{0}-2=0$ must have at least one positive and real root. Hence singularity arising in this case is naked, it would imply the existence of outgoing radial null geodesics emanating from the singularity [9]. If equation (29) does not have real and positive root then gravitational collapse ends into black hole, it means the singularity is covered in an event horizon.

Numerical calculation shows that this equation has at least one real positive root. In particular, if we take $\mu^{2}=0.01, \delta^{2}=0.01, \lambda=0.1$ and $a=0.1$ then positive root of equation (29) is $X_{0}=7.4645$. This shows that the gravitational collapse ends into a naked singularity.

We shall consider some different values of $\mu^{2}, \delta^{2}, \lambda$ and $a$ to study the nature of singularity of equation (29) For fixed $\mu^{2}=0.01$ and $\delta^{2}=0.01$ then equation (29) reduces to the form

$$
(0.01+0.01) X_{0}^{3}-\lambda X_{0}^{2}-a X_{0}-2=0
$$

then the values of $X_{0}$ obtained from the equation (30) for different values of $\lambda$ and $a$ in non-spherical gravitational collapse of radiating dyon solution with monopole field are given in the following table.

Table 1: Values of $X_{0}$ for different values of $\lambda$ and $a$

\begin{tabular}{|r|r|r|r|r|r|}
\hline \multirow{2}{*}{\multicolumn{1}{|c|}{$\lambda$}} & \multicolumn{5}{|c|}{$X_{0}$} \\
\cline { 2 - 6 } & $\mathrm{a}=0.1$ & 0.25 & 0.5 & 0.75 & 0.85 \\
\hline 0.1 & 7.4645 & 8.0792 & 9.0078 & 9.8424 & 10.1549 \\
\hline 0.2 & 11.2369 & 11.7814 & 12.6111 & 13.3655 & 13.6502 \\
\hline 0.3 & 15.7225 & 16.1568 & 16.8375 & 17.4736 & 17.7173 \\
\hline 0.4 & 20.4825 & 20.8305 & 21.3875 & 21.919 & 22.1252 \\
\hline 0.5 & 25.3528 & 25.6396 & 26.1044 & 26.554 & 26.7299 \\
\hline 0.6 & 30.2743 & 30.517 & 30.9134 & 31.3001 & 31.4523 \\
\hline 0.7 & 35.2226 & 35.4324 & 35.7769 & 36.115 & 36.2486 \\
\hline 0.8 & 40.1863 & 40.371 & 40.6751 & 40.9748 & 41.0934 \\
\hline 0.9 & 45.1598 & 45.3245 & 45.5964 & 45.8652 & 45.9718 \\
\hline
\end{tabular}

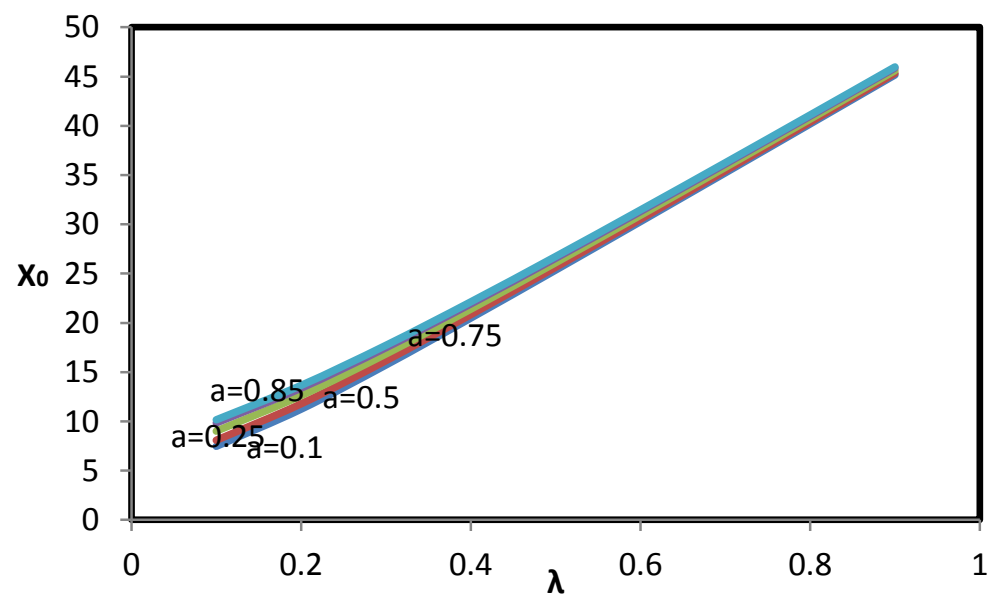

Fig. 1: Graph of the values of $X_{0}$ against the value of $\lambda$. 
Effect of Monopole Field on the Non-Spherical Gravitational Collapse of Radiating Dyon Solution.

From the graph we may observe that the values of $X_{0}$ increases as increase the value of $\lambda$. Also we note that the lower value of $X_{0}$ shifted to the peak. It is observing that when increase monopole value the root of equation increases.

For fixed $a=0.25$ and $\delta^{2}=0.01$ then equation (29) reduces to the form

$$
\left(\mu^{2}+0.01\right) X_{0}^{3}-\lambda X_{0}^{2}-0.25 X_{0}-2=0
$$

then the values of $X_{0}$ obtained from the equation (31) for different values of $\lambda$ and $a$ in non-spherical gravitational collapse of radiating dyon solution with monopole field are given in the following table.

Table 2: Values of $X_{0}$ for different values of $\lambda$ and $\mu^{2}$

\begin{tabular}{|r|r|r|r|r|r|}
\hline \multirow{2}{*}{$\lambda$} & \multicolumn{5}{|c|}{$X_{0}$} \\
\cline { 2 - 6 } & $\mu^{2}=0.01$ & $\mu^{2}=0.02$ & $\mu^{2}=0.03$ & $\mu^{2}=0.04$ & $\mu^{2}=0.05$ \\
\hline 0.1 & 8.0792 & 6.3206 & 5.3849 & 4.7886 & 4.3678 \\
\hline 0.2 & 11.7814 & 8.5525 & 6.9391 & 5.9633 & 5.3039 \\
\hline 0.3 & 16.1568 & 11.2651 & 8.8456 & 7.4048 & 6.4479 \\
\hline 0.4 & 20.8305 & 14.2467 & 10.9835 & 9.0422 & 7.7577 \\
\hline 0.5 & 25.6396 & 17.3675 & 13.256 & 10.8053 & 9.1824 \\
\hline 0.6 & 30.517 & 20.5629 & 15.6058 & 12.6455 & 10.6822 \\
\hline 0.7 & 35.4324 & 23.8011 & 18.0015 & 14.5334 & 12.2302 \\
\hline 0.8 & 40.371 & 27.0656 & 20.4258 & 16.4517 & 13.8098 \\
\hline 0.9 & 45.3245 & 30.347 & 22.8689 & 18.3902 & 15.4107 \\
\hline
\end{tabular}

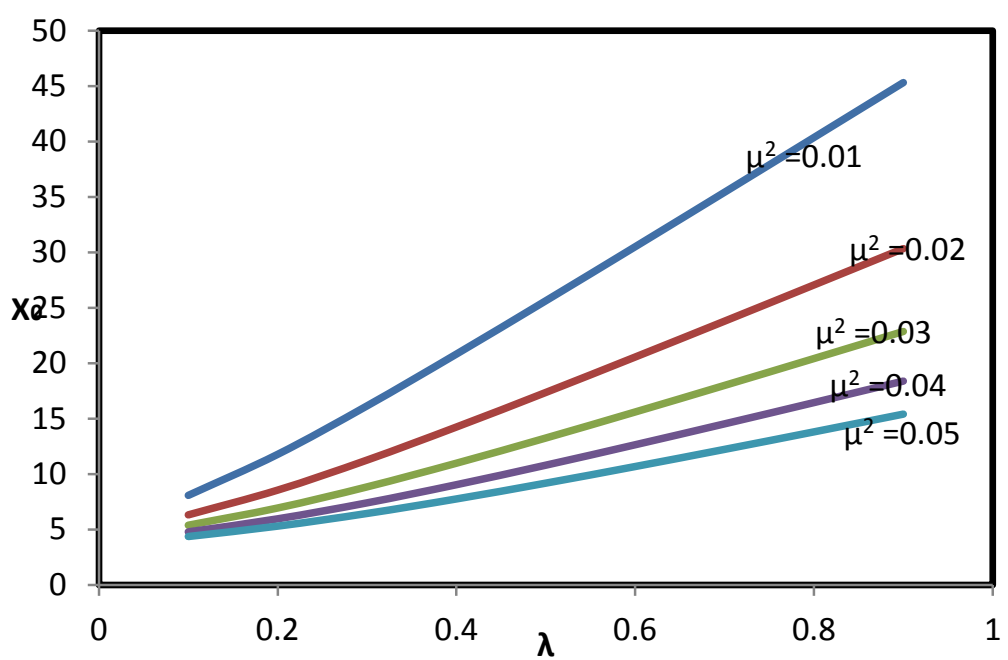

Fig. 2: Graph of the values of $X_{0}$ against the value of $\lambda$.

From the graph it is seen that the initially the values of $X_{0}$ are closest for different values of magnetic charge and which are diffracted for higher and higher values of $\lambda$.

For fixed $\lambda=0.1$ and $\delta^{2}=0.01$ then equation (29) reduces to the form

$$
\left(\mu^{2}+0.01\right) X_{0}^{3}-0.1 X_{0}^{2}-a X_{0}-2=0
$$

then the values of $X_{0}$ obtained from the equation (32) for different values of $\lambda$ and $a$ in non-spherical gravitational collapse of radiating dyon solution with monopole field are given in the following table.

Table 3: Values of $X_{0}$ for different values of $a$ and $\mu^{2}$

\begin{tabular}{|r|r|r|r|r|r|}
\hline \multirow{2}{*}{$\mu^{2}$} & \multicolumn{5}{|c|}{} \\
\cline { 2 - 6 } & $\mathrm{a}=0.1$ & 0.25 & 0.5 & 0.75 & 0.85 \\
\hline 0.01 & 7.4645 & 8.0792 & 9.0078 & 9.8424 & 10.1549 \\
\hline 0.02 & 5.8507 & 6.3206 & 7.0434 & 7.7026 & 7.9512 \\
\hline 0.03 & 5 & 5.3849 & 5.9847 & 6.5378 & 6.7475 \\
\hline 0.04 & 4.4597 & 4.7886 & 5.3057 & 5.7867 & 5.9699 \\
\hline 0.05 & 4.0788 & 4.3678 & 4.8253 & 5.2537 & 5.4174 \\
\hline 0.06 & 3.7921 & 4.0511 & 4.4632 & 4.8512 & 5 \\
\hline
\end{tabular}




\begin{tabular}{|l|l|l|l|l|l|}
0.07 & 3.5662 & 3.8017 & 4.1781 & 4.5339 & 4.5339 \\
\hline 0.08 & 3.3822 & 3.5988 & 3.9461 & 4.2757 & 4.2757 \\
\hline 0.09 & 3.2285 & 3.4295 & 3.7526 & 4.0603 & 4.0603 \\
\hline
\end{tabular}

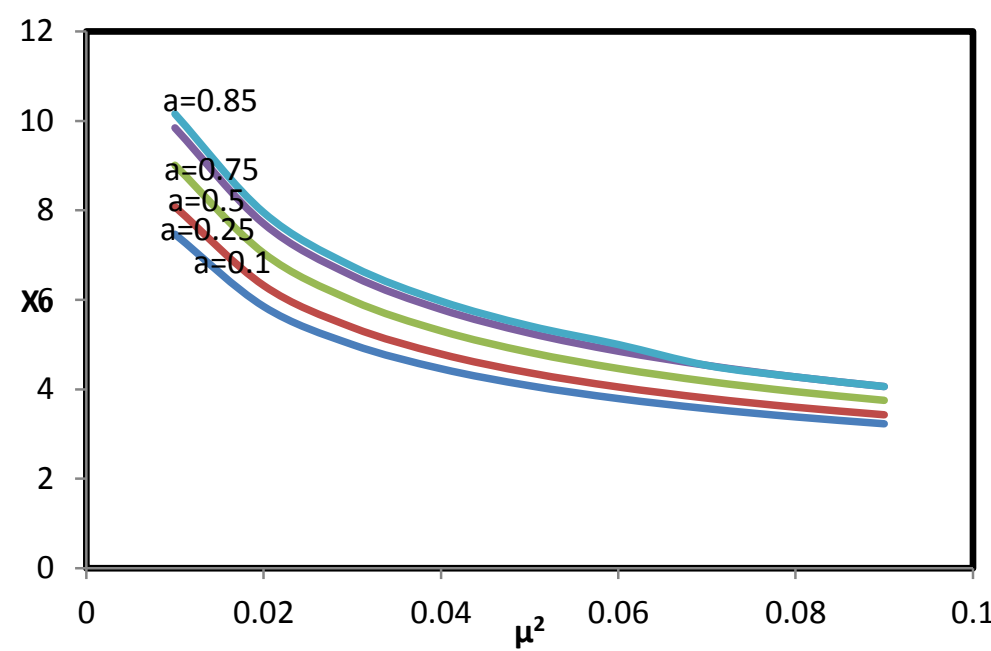

Fig. 3: Graph of the values of $X_{0}$ against the value of $\mu^{2}$.

The graph shows that the values of $X_{0}$ is at peak then shifted towards the lower value for increasing the value of magnetic charge, i.e magnetic charge affects the roots of equation

\section{Strength Of Singularity}

It was seen the nakedness of the singularity, we now look towards strength of the singularity. The strength of the singularity is an important issue because there have been attempt to relate it to the stabilility [25]. According to the Clark and Krolak [26], a sufficient condition for a singularity is to be strong in the sense of Tripler [27], if at least one radial null radial null geodesic with affine parameter $k$, limit at $k \rightarrow 0$ at the singularity.

$$
\Psi=\lim _{k \rightarrow 0} k^{2} R_{i j} k^{i} k^{j}>0,
$$

where $k^{i}$ is the tangent to the null geodesics and $R_{i j}$ is the Ricci tensor.

In the present paper we find that

$$
\begin{aligned}
\Psi & =\lim _{k \rightarrow 0} k^{2} R_{i j} k^{i} k^{j}=\lim _{k \rightarrow 0} k^{2} \frac{q \dot{m}}{r^{2}}\left(k^{v}\right)^{2} \\
& =\left[\lambda-\left(2 \delta^{2}+2 \mu^{2}\right) X\right] \quad \lim _{k \rightarrow 0}\left(\frac{k r}{r^{2}}\right)^{2}
\end{aligned}
$$

Using the fact that the singularity is approached, $k \rightarrow 0, r \rightarrow 0$ and $X \rightarrow X_{0}$, we find that

$$
\Psi=\frac{\left[\lambda-\left(2 \delta^{2}+2 \mu^{2}\right) X\right]}{\left[-a-\lambda X_{0}+\left(\delta^{2}+\mu^{2}\right) X_{0}^{2}\right]}
$$

For our particular case ( i. e. $\lambda=0.1, \mu^{2}=0.01, \delta^{2}=0.01, a=0.25$ and $X_{0}=8.0792$ ) we have $[\lambda$ $\left.\left(2 \delta^{2}+2 \mu^{2}\right) X\right]<0$, hence the naked singularity arising in the non-spherical gravitational collapse of radiating Dyon solution with monopole field is not a strong curvature singularity. i.e. weak curvature singularity is found.

\section{Concluding Remarks}

The gravitational collapses of toroidal (planar or cylindrical) models are not only astrophysically interesting but also important to a better understanding of these conjectures. Naked singularities are worth studying in classical physics and in some form of quantum gravity, as the appearance of extremely strong curvature is in principle observable.

In the present work we have shown that the naked singularities are formed in the gravitational collapse of non-spherical monopole radiating Dyon solution. It is pointed out that electric charge or magnetic charge supports the naked singularity. It has been shown that in ref. 24 under some condition on mass function spherical gravitational collapse of radiating Dyon solution yields a naked singularity. Hence for $\mathrm{CCH}$ point of view one would like to know whether the gravitational collapse of radiating dyon solution preserve this characteristic when one makes transition from spherical to non-spherical case. keeping this view, we have analyse the collapse of radiating Dyon solution in non-spherical symmetric spacetimes with monopole field and found that gravitational collapse of these models ends into a naked singularity. 
In this paper we observe an interesting feature that the naked singularity arising in non-spherical gravitational collapse of monopole radiating Dyon solution is weak curvature singularity, it means the singularity found to be globally naked, that is monopole field affects the strength of singularity.

\section{References}

[1] D M Eardley and L Smarr, Time function in numerical relativity: Marginally bound dust collapse, Phys. Rev,. D 19, 1979, 22392259.

[2] D Christodoulou, Violation of cosmic censorship in gravitational collapse of a dust cloud, Commun. Math. Phys., 93, 1984, 171195.

[3] R P A C Newman, Strength of naked singularities in Tolman-Bondi space-times, Class. Quantum Grav., 3, $1986,527$.

[4] K D Patil and U S Thool, Spherical and non-spherical gravitational collapse in Husain space-time, Int. J. Mod. Phys., D 14(6), 2005, 873-882.

[5] K D Patil, Structure of radial null geodesics in higher-dimensional dust collapse, Phys. Rev. D 67, $2003,024017$.

[6] P S Joshi, Key problem in black hole physics today, Bull.Astr. Soc. India 39, 2011, 1-20.

[7] P S Joshi, Spacetime singularities and cosmic censorship, arXiv: gr-qc: 1010.2049 V1, 2010.

[8] P S Joshi, Key problem in black hole physics today, arXiv: gr-qc: 1104.3741 V 1, 2011.

[9] K D Patil and U S Thool, Spherical and non-spherical gravitational collapse in monopole anti- de-sitter vaidya space time, Int. J. Mod. Phys. D 15(11), 1977(2006).

[10] K D Patil S S Zade and A N Mohod, Gravitational collapse of radiating Dyon solution and cosmic censorship hypothesis, Chin. Phys. Lett. vol 23, 2008, 854.

[11] R Penrose, Gravitational collapse: The role of general relativity, Riv. Nuovo Cimento 1, 1969, 252.

[12] J R Oppenheimer and H Snyder, On continued gravitational contraction, Phys. Rev. 56, 1939, 455.

[13] G Lemaitre, Ann. Soc. Sci. Bruxelles, A53, 1933, 51.

[14] TomohiroHarada, Gravitational collapse and naked singularities, Pramana journal of physics, Vol. 63, 2004, 741-753.

[15] R Penrose, in General relativity, an Einstein centenary survey edited by S W Hawking and W Israel (Cambridge University Press, Cambridge, England, 1979) p. 581

[16] P S Joshi, Spacetime singularities, arXiv: gr-qc: $1311.0449, \mathrm{~V} 1,2013$

[17] Victor Cardoso, Oscar J C Dias and J P S Lemos, Phys. Rev. D 67, 2003, 064026.

[18] Anzhong Wang, Generalized Vaidya solution, Gen, Relativ. Grav. 31, 1999 (1).

[19] J P S Lemos, Phys. Rev. D 59, 1999, 044020.

[20] Anzhong Wang and Yumei Wu, Generalized Viadya solutions, Gen. Relativ. Gravit. Vol. 31, No.1, 1999, $107-114$.

[21] S W Hawking and G F R Ellis, Cambridge university Press, Cambridge, (1973).

[22] P S Joshi, Global aspects in gravitation and cosmology, clarendon press, oxford, (1993).

[23] S G Ghosh and N Dadhich, Gravitational collapse of null strange quark fluid and cosmic censorship, arXiv: gr-qc/0211019, V 1, 2002.

[24] S G Ghosh and N Dadhich, On naked singularities in higher dimensional Vaidya spacetimes, arXiv: gr-qc/0105085 V 2, 2001.

[25] S S Deshingkar, P S Joshi and I H Dwivedi, Physical nature of central singularity in spherical collapse, Phys. Rev. D 59, 1999, 044018 .

[26] C J S Clark and A Krolak, J. Geo. Phys., 2, 1986, 127.

[27] F J Tripler, singularities in conformally flat spacetime, Phys. Lett. A 64, 1977(8). 